psychopraxis. neuropraxis $2018 \cdot 21: 195$ https://doi.org/10.1007/s00739-018-0527-6

(c) Springer-Verlag GmbH Austria, ein Teil von Springer Nature 2018

CrossMark

\section{Martin Aigner}

Abteilung für Erwachsenenpsychiatrie, Universitätsklinik für Psychiatrie und Psychotherapie, Karl Landsteiner Privatuniversität für Gesundheitswissenschaften, Universitätsklinikum Tulln, Tulln, Österreich

\title{
Internationale Klassifikation psychischer Störungen
}

Liebe Leserinnen und Leser der psychopraxis.neuropraxis!

Das ICD-11 ist schon lange erwartet worden, nachdem das DSM-5 bereits in der deutschen Version seit 3 Jahren am Markt ist. Am 18.06.2018 war es soweit: Die WHO hat die 11 . Version des ICD (International Classification of Diseases) vorgestellt (http://www.who. int/classifications/icd/en/). Das „Kapitel V (F), die Internationale Klassifikation psychischer Störungen" des ICD-10 wurde im Wesentlichen in das Kapitel „06 Psychische, Verhaltens- oder neurologische Entwicklungsstörungen “ des ICD-11 übergeführt.

Die „nichtorganischen Schlafstörungen (F51)“ wurden in einem gemeinsamen Kapitel „07 Schlaf-Wachstörungen“ mit den organischen Schlafstörungen zusammengefasst. Das Kapitel „F52 nichtorganische sexuelle Funktionsstörungen“ ist ebenso wie das Kapitel „F64 Störungen der Geschlechtsidentität“ im Kapitel „08 Zustände in Zusammenhang mit sexueller Gesundheit" aufgegangen. Die Struktur der Klassifikation wurde vom ICD-10 bis zum ICD-11 deutlich verändert. War im ICD-10 noch das „triadische System“ implizit vorhanden, ist im ICD-11 eine neue Klassifikationslogik ähnlich wie im DSM-5 angewendet worden.

\section{Zu den Themen in diesem Heft}

Gerade in der Medizin sind interkulturelle Teams zur Norm geworden. Dennoch braucht es besondere Kompetenzen, damit diese Teams gut funktionieren können. In diesem Heft wird die Zusammenarbeit in interkulturellen Teams thematisiert. Dabei werden mögliche Miss- verständnisse in multikulturellen Teams aufgezeigt und Lösungsvorschläge präsentiert. Die Psychotherapie wird gleich in 2 Artikeln zum Thema gemacht: Zum einen wird die Bedeutung der psychotherapeutischen Gruppentherapien anhand eines Falles dargestellt. Zum anderen wird eine Studie zur Psychotherapieausbildung in Österreich vorgestellt. Dies zeigt welche Bedeutung die Psychotherapie in der "psychiatrischen Praxis“ gewonnen hat. In 2 weiteren Fallbeispielen, einem psychiatrischen und einem neurologischen Fall, werden die diagnostischen Grenzen der Epilepsie ausgelotet. Das Wissen um die Epilepsie ist in den letzten Jahrzehnten enorm gestiegen, dennoch sind viele Facetten der Epilepsie noch nicht geklärt. Fallberichte können dazu beitragen, die Komplexität dieser Störung aufzuzeigen. Besonders freut es mich, dass dies nicht nur von neurologischer Seite kommt, sondern auch aus dem psychiatrischen Bereich. Ein weiterer Beitrag ist der Rolle des Vitamin D bei der diabetischen Neuropathie gewidmet. Dieser Artikel befasst sich kritisch und umfassend mit der Möglichkeit, Vitamin D in der Prophylaxe der diabetischen Neuropathie einzusetzen. Auch die Sexualität kommt nicht zu kurz: Mit der Objektophilie wird eine noch nicht klassifizierte Spielart der Liebe zu Objekten beschrieben. Ein Hinweis darauf, dass sich die Sexualität immer mehr von der Fortpflanzung und menschlichen Beziehungen zu entfernen scheint.

Viel Freude und neue Erkenntnisse beim Lesen dieses Heftes wünscht Ihnen, Ihr

Martin Aigner

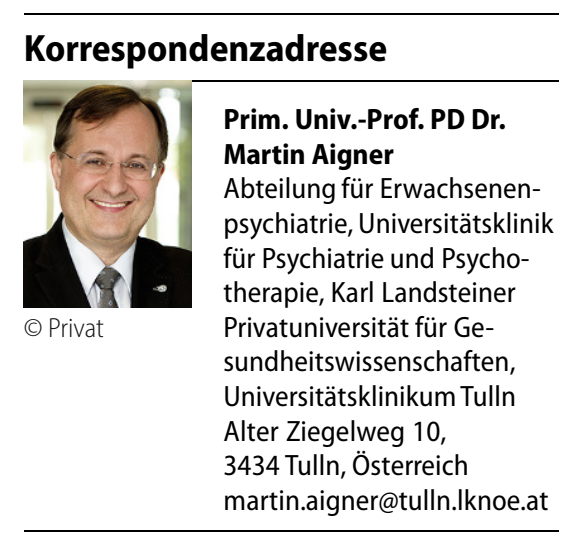

Interessenkonflikt. M. Aigner gibt an, dass kein Interessenkonflikt besteht. 